\title{
Study on the Single Toxicity of Five Azole Fungicides for Green Algae
}

\author{
Yilun Dai ${ }^{1,2,3,4,5, *}$, Jianglong Shen ${ }^{1,2,3,4,5}$, Yutong Sun ${ }^{1,2,3,4,5}$ and Yanan Li 1, 2, 3, 4, 5 \\ ${ }^{1}$ Shaanxi Provincial Land Engineering Construction Group Co., Ltd. China \\ ${ }^{2}$ Institute of Land Engineering and Technology, Shaanxi Provincial Land Engineering Construction Group Co., Ltd. China \\ ${ }^{3}$ Key Laboratory of Degraded and Unused Land Consolidation Engineering, Ministry of Natural Resources China \\ ${ }^{4}$ Shaanxi Provincial Land Consolidation Engineering Technology Research Center China \\ ${ }^{5}$ Land Engineering Technology Innovation Center, Ministry of Natural Resources China
}

\begin{abstract}
Azole fungicides are widely used to control diseases and insect pests of vegetables, fruits and flowers because of their good antibacterial effect and regulating plant growth. However, their extensive use inevitably enters the water environment with rainfall and surface runoff, which leads to potential harm to aquatic organisms. At present, the research on the biological toxicity of azole fungicides mainly focuses on single azole compounds, such as Paclobutrazol, hexaconazole, propiconazole and Difenoconazole. However, the systematic study on the toxic effects of multi-component mixtures of azole fungicides is still very lacking, and it is unable to accurately assess the ecological risk. Therefore, in this paper, five common azole fungicides (imidazole, tebuconazole, triadimefon, tricyclazole and hymexazol) in the environment were taken as target pollutants. Scenedesmus obliquus was used as the toxicity indicator. The mixture system was designed by the equal effect concentration ratio method and linear average ray method. Study the $96 \mathrm{~h}$ toxicity and interaction of Scenedesmus obliquus under combined stress of azole fungicides. The main research results are as follows:The $96 \mathrm{~h}$ toxicity test of five typical azole fungicides to Scenedesmus obliquus was determined by microplate toxicity analysis method. Fitting with Weibull function and taking EC50 as the criterion, the results show that the single toxicity order of the five tested fungicides was:tebuconazole $>$ Triadimefon $>$ oxacillin $>$ Tricyclazole $>$ imidazole.
\end{abstract}

Key words: azole fungicides, Scenedesmus obliquus, toxicity.

\section{Introduction}

Fungicides usually refer to chemical preparations that can effectively control or kill microorganisms such as bacteria, fungi and algae in the water system environment [1], and generally in the international arena, can be used as agents for the control of various pathogenic microorganisms, known as as a fungicide. The early fungicides were mainly inorganic fungicides, such as Bordeaux mixture, the world's earliest inorganic fungicides. In 1914, Germany took the lead in using organic mercury compounds to control wheat smut $[2,3]$, which marked the beginning of the development of organic fungicides [4]. In 1944, Woolley successfully synthesized the first azole fungicide, benzimidazole. During this period, after more than 70 years of development [5], azole fungicides have gradually become the protagonist of the sterilization market, and are widely used in agricultural production and personal skin care products. and medical drugs. In agriculture, the production of a large number of food crops is reduced every year due to fungal infection, and sometimes even threatens human health. For example,
Fusarium can produce mycotoxins when plants grow. The use of azole fungicides can not only prevent the infection of crops, but also can prevent the infection of crops. Infected crops can be effectively treated [6-11]. By 2017, more than 60 azole pesticides have been sold worldwide, accounting for $25 \%$ of the global application of fungicides [12]. In terms of personal skin care products, azole fungicides are widely used in household products such as shampoos, skin care products, soaps, toothpastes and shower gels due to their low toxicity, broad spectrum and high efficiency [13], such as ketoconazole It is often used in anti-dandruff hair care formulations [14]. Cribazole is not only used as an anti-dandruff active ingredient, but also as an anti-fungal preservative and anti-aging agent, and its content is up to $2 \%$ in washing products. The highest in washing products is $0.5 \%$, and the highest in cosmetic products is $0.5 \%$ [14]; the annual sales volume in my country exceeds 3,800 tons [15]. In medical terms, of the 2 million fungi known to date, about 600 are known as human fungal pathogens, of which $3 \%$ to $4 \%$ can cause invasive fungal infections [16]. Over the past 30 years, annual Invasive fungal infections kill between 1 and 2

\footnotetext{
*Corresponding author: 373289543@qq.com
} 
million people, and the mortality rate far exceeds that of malaria and tuberculosis [17]. Among the more than 40 brands of antifungal drugs clinically used on the market, azole fungicides are considered to be the most It has the best curative effect and the most widely used fungicides [18-21].

Chemical pollutants in the actual natural environment often do not exist alone, but in the form of mixtures. With the vigorous development of industry, agriculture, and manufacturing, the types of pollutant mixtures are increasing, and new types of pollutants are increasing. The ecological and environmental problems caused by them are not optimistic. Therefore, in the past half century, the research on the combined toxicity of mixtures has become more and more Having attention. For example, on the issue of industrial sewage treatment, the possibility that there are still unclean pollutants in the treated industrial sewage cannot be excluded. Various new types of pollutants are increasing day by day, and their toxicity is rare, and their detection cannot be analyzed and expressed by a single physical and chemical index. On the other hand, biological monitoring can more vividly reflect the pollution and toxicity of pollutants in different situations in a certain area.

In order to reveal the ecotoxicity of azole fungicides exposed in the natural environment, this study took five azole fungicides as target pollutants, and investigated the effects of azole fungicides at different concentrations on the growth of the toxicity indicator organism, Scenedesmus obliquus. To clarify the single toxicity of these five typical azole fungicides to Scenedesmus obliquus, and to provide basic toxicity data for the risk assessment of mixed pollutants in the water environment.

\section{Matrials and methods}

\subsection{Experimental Materials}

\subsubsection{Experimental Reagents}

Five typical azole fungicides were selected in this paper as the research target pollutants. The specific physical and chemical properties are shown in Table 1, and the chemical structure is shown in Figure 1.

Table 1 Physicochemical properties of 5 target pollutants

\begin{tabular}{|c|c|c|c|c|c|c|}
\hline $\begin{array}{c}\text { Serial } \\
\text { Number }\end{array}$ & Compound & $\begin{array}{l}\text { Short } \\
\text { Name }\end{array}$ & CAS & $\begin{array}{c}\text { Relative } \\
\text { molecular } \\
\text { mass }\end{array}$ & $\begin{array}{c}\text { Purity } \\
(\%)\end{array}$ & Source \\
\hline 1 & Imidazole & IDZ & $\begin{array}{l}288- \\
32-4\end{array}$ & 68.08 & 99.50 & Accustandard \\
\hline 2 & Tebuconazole & $\mathrm{CDT}$ & $\begin{array}{c}80443- \\
41-0\end{array}$ & 307.82 & 98.70 & $\begin{array}{c}\text { Dr. } \\
\text { Ehrenstorfer } \\
\text { GmbH }\end{array}$ \\
\hline 3 & Triadimefon & TDF & $\begin{array}{c}43121- \\
43-3\end{array}$ & 293.75 & 99.60 & Accustandard \\
\hline 4 & Tricyclazole & $\mathrm{TCL}$ & $\begin{array}{c}41814- \\
78-2\end{array}$ & 189.24 & 99.50 & Accustandard \\
\hline 5 & Oxymetholone & MHZ & $\begin{array}{c}10004- \\
44-1\end{array}$ & 99.09 & 99.00 & $\begin{array}{c}\text { Dr. } \\
\text { Ehrenstorfer } \\
\text { GmbH }\end{array}$ \\
\hline
\end{tabular}

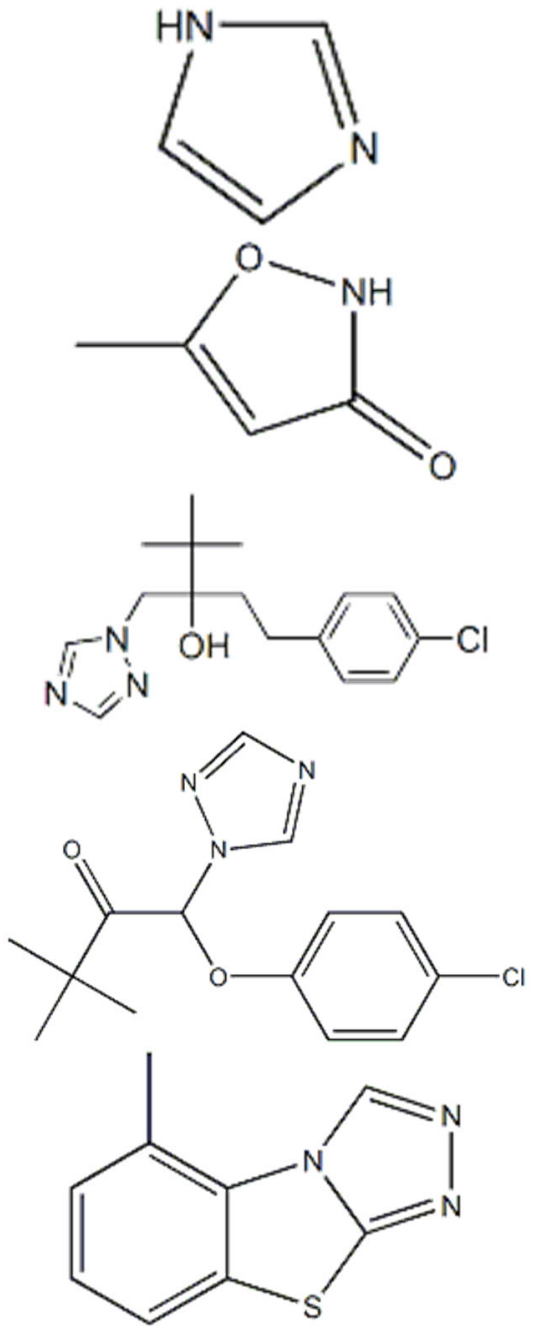

Figure 1. Chemical Structures of Five Azole Fungicides

\subsubsection{Algae Species}

Scenedesmusobliquus were purchased from the Freshwater Algae Seed Bank (FACHB) of the Type Culture Collection Committee of the Chinese Academy of Sciences, and the serial numbers were FACHB-12. After receiving the algae seed, transfer the algae seed to the prepared medium for cultivation in the clean bench.

\subsubsection{Cultivation of Algae}

The BG11 medium was used to culture Scenedesmus obliquus, and the medium was sterilized in a sterilizing pot at $121{ }^{\circ} \mathrm{C}$ and $101 \mathrm{KPa}$ for $20 \mathrm{~min}$. After cooling, green algae were aseptically inoculated in the ultra-clean workbench, and then placed in an artificial constant temperature light incubator for cultivation. The set temperature was $22^{\circ} \mathrm{C}$, the illuminance was about 2000lux, and the light-dark cycle was $12 \mathrm{~h}: 12 \mathrm{~h}$. Expand the culture every $5-7 \mathrm{~d}$, that is, dilute the transfer algae at a ratio of $1: 2$, so that the algae can fully enter the logarithmic growth phase. The most vigorous period of algal cell metabolism is at $8-10 \mathrm{am}$, so it is the best time for algal species transfer [66]. The algae should be cultivated in a sterile environment throughout the process. Before the 
toxicological test, the algal seeds in the logarithmic growth phase were transferred to fresh medium for cultivation, and the experiment was carried out after culturing for 2 days until the initial light absorption value of the algal seeds was $0.22-0.25$. In order to avoid the experimental error caused by the volatilization of the drug, a transparent cover plate should be added during the experimental exposure period.

\subsection{Toxicity Experiment}

\subsubsection{Green Algae Acute Microplate Experiment}

The algal microplate toxicity test uses a standard transparent 96-well microplate, and the microplate sample loading design method is as follows.

First, add $200 \mu \mathrm{L}$ of ultrapure water to the four sides of the 96-well microplate (36 wells in total) to prevent edge effects; $100 \mu \mathrm{L}$ of ultrapure water was added to the 24 wells in columns 2, 6, 7 and 11 respectively as blank control; A total of 6 wells in the 3rd column and 6 wells in the 8th column were respectively added with different concentrations of pesticide pollutants designed according to the dilution factor (see Equation 2.1, and supplemented with ultrapure water to make the volume $100 \mu \mathrm{L}$; The parallel trials for column 3 are columns 4 and 5, and the parallel trials for column 8 are columns 9 and 10; Finally, $100 \mu \mathrm{L}$ of algae liquid with an optical density value of about 0.15 was added to the middle 60 wells, so that the total volume of each well was $200 \mu \mathrm{L}$; After adding samples to the microplate, cover with a transparent cover, and place it in a biochemical incubator with a temperature of $22^{\circ} \mathrm{C}$, an illuminance of 2000 lux, and a light-dark ratio of $12 \mathrm{~h}: 12 \mathrm{~h}$. At $96 \mathrm{~h}$, the microplate was placed in a spectrophotometer to measure the optical density (OD) at a wavelength of $681 \mathrm{~nm}$. The microplate was repeated at least 3 times.

$$
F=\left(\frac{C_{l}}{C_{H}}\right)^{\frac{1}{n-1}}, \mathrm{n}=1,2 \ldots \ldots
$$

Among them, $\mathrm{CH}$ and $\mathrm{CL}$ are the highest and lowest concentrations of the experiment, respectively, and $n$ represents the number of concentration gradients in the range of $\mathrm{CH}$ and $\mathrm{CL}$. Then the twelve concentration points are $\mathrm{Cn}=\mathrm{C} 0 * \mathrm{Fn}, \mathrm{n}=1,2 \ldots$ Among them, $\mathrm{C} 0$ is the concentration of the stock solution, and $\mathrm{Cn}$ is the set concentration. In this paper, the growth inhibition rate (I) of pollutants to Scenedesmus obliquus is used as the toxicity index of the microplate test, and the calculation formula of $\mathrm{I}$ is as follows: $\mathrm{I}=(\mathrm{ODt}, \mathrm{i}-\mathrm{ODt}, 0) /(\mathrm{OD} 0, \mathrm{i}-$ OD0,0), where ODti is the OD value of the pollutant experimental group at the $\mathrm{i}$-th time, and the OD value of the OD0i blank control group.

\subsubsection{Data Processing}

The experimental toxicity data were processed by the MATLAB program written by Qin Litang, and the graph of the fitted curve was drawn by the Origin software. To derive effect sizes and effect concentrations for individual compounds and mixtures, a nonlinear fit to the observed concentration-response data must be performed. For classical S-type CRC, Logit or Weibull nonlinear function is generally used for fitting. The function expression is shown in Table 2., where I represents the effect, c represents the concentration of a single compound or mixture, $\alpha$ is the position parameter, and $\beta$ is the slope parameter [23]. The Logit function and the Weibull function are classical two-parameter functions. The Logit function is based on the integral statistics of logistic distribution and takes half effect $(50 \%)$ as the center symmetry, so it is suitable for fitting a symmetrical curve. The Weibull function, originally proposed by Gompertz, is based on extreme value distribution integral statistics and is suitable for most asymmetric concentration-effect curves. The MTA method used in this study has only 12 concentration gradients, and the two-parameter Logit and Weibull functions should be used for nonlinear fitting [24].

Table 2. Two Common S-Type Concentration-Effect Curve Fitting Models

\begin{tabular}{cc}
\hline Function type & Analysing way \\
\hline Logit & $\mathrm{I}=1 /(1+\exp (-\alpha-\beta \operatorname{lgc}))$ \\
Weibull & $\mathrm{I}=1-\exp (-\exp (\alpha+\beta \operatorname{lgc}))$ \\
\hline
\end{tabular}

\section{Results}

Table 3. Concentration-effect fitting curve parameters of five azole fungicides on Scenedesmus obliquus

\begin{tabular}{ccccccc}
\hline Compound & Fitfunction & $\alpha$ & $\beta$ & R2 & RMSE & $\begin{array}{c}\text { EC50 } \\
\left(\begin{array}{c}\text { (mol·L- } \\
1)\end{array}\right.\end{array}$ \\
\hline IDZ & Weibull & 5.706 & 2.200 & 0.987 & 0.0393 & $2.20 \mathrm{E}-03$ \\
CDT & Weibull & 6.446 & 1.509 & 0.991 & 0.0242 & $3.22 \mathrm{E}-05$ \\
TDF & Weibull & 4.966 & 1.331 & 0.975 & 0.0339 & $9.81 \mathrm{E}-05$ \\
TCL & Weibull & 3.174 & 1.175 & 0.977 & 0.0287 & $8.86 \mathrm{E}-04$ \\
HMZ & Weibull & 5.827 & 1.708 & 0.992 & 0.0299 & $2.55 \mathrm{E}-04$ \\
\hline
\end{tabular}
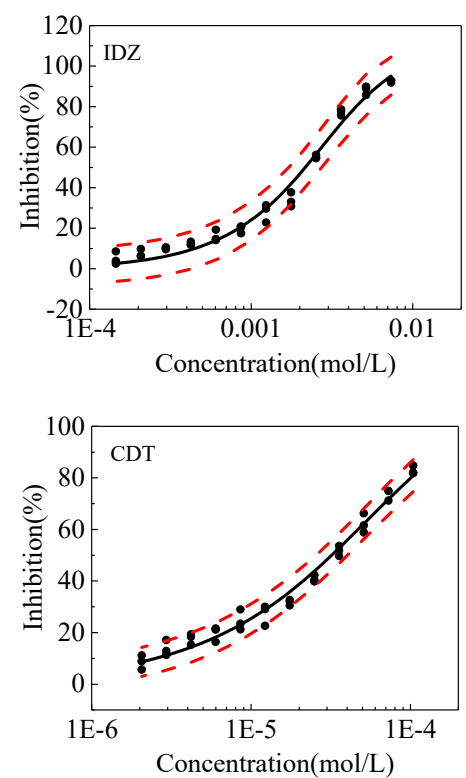

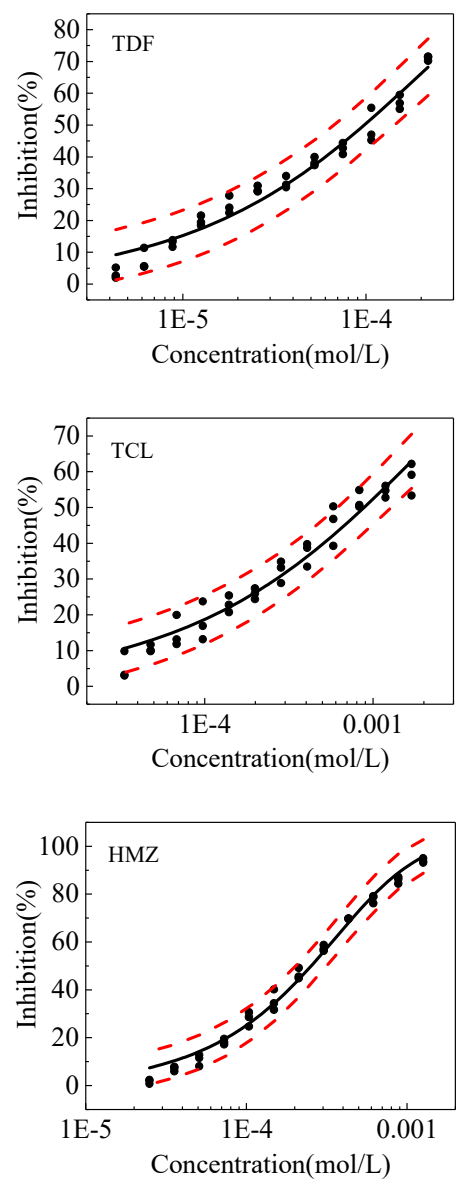

Figure 2 Concentration-response curves (CRC) of five azole fungicides on Scenedesmus obliquus

The biological toxicity of azole fungicides was tested with Scenedesmus obliquus as the indicator organism, and the toxicity of azole fungicides was expressed by the inhibition rate of Scenedesmus obliquus at 96 hours. The concentration-effect data of the five azole fungicides on Scenedesmus obliquus can be fitted by the Weibull function or the Logit function. The fitting model parameters (regression parameters $\alpha$ and $\beta$ values, statistical parameters R2 and RMSE) are shown in Table 2. The $R 2$ values of the five azole fungicides on the toxicity data of Scenedesmus obliquus were between 0.975 and 0.992 , all greater than 0.95 ; The RMSE values of the fitted curves ranged from 0.024 to 0.0339 , all less than 0.028 , which indicated that the fitting results of the Weibull model were accurate and reliable. Figure 2. shows the concentration-response number fitted curves (CRCs) of the five azole fungicides, all showing a classic $\mathrm{S}$ shape. From Table 3, the toxicity under $50 \%$ effect can be obtained as: tebuconazole $>$ Triadimefon $>$ oxacillin $>$ Tricyclazole $>$ imidazole.

\section{References}

1. Liuchen Zhao. Bactericidal properties of bisdecyl dimethyl ammonium formate and its composite system[D]. China Daily Chemical Industry Research Institute, 2017.
2. Large, E. C. Control of Potato Blight (Phytophthora infestans) by Spraying with Suspensions of Metallic Copper[J]. Nature, 1943, 151(3820): 195-206.

3. Melnikov N N, Gunther F A, Gunther J D. Chemistry of pesticides[M]. Residue Reviews, 1971, 36(1).

4. Klittich C J. Milestones in Fungicide Discovery: Chemistry that Changed Agriculture[J]. Plant Health Progress, 2008, 9(1):1-8.

5. Woolley D W, Pringle A. Relationship of chemical structure to antibacterial activity among analogues of dimethyldiaminobenzene[J]. Journal of Biological Chemistry, 1952, 194(2): 729-746.

6. Janna H, Scrimshaw M D, Williams R J, et al. From Dishwasher to Tap Xenobiotic Substances Benzotriazole and Tolyltriazole in the Environment[J]. Environmental ence \& Technology, 2011, 45(9): 3858-3864.

7. H., Dooley, M., et al. Effect of azole fungicide mixtures, alternations and dose on azole sensitivity in the wheat pathogenZymoseptoria tritici[J]. Plant Pathology, 2015.65(1):124-136.

8. Giavini E, Menegola E. Are azole fungicides a teratogenic risk for human conceptus[J].Toxicology Letters, 2010, 198(2): 106-111.

9. Brauer V S, Rezende C P, Pessoni A M, et al. Antifungal Agents in Agriculture: Friends and Foes of Public Health[J]. Biomolecules, 2019, 9(10): 521534.

10. Escher B I, Baumgartner R, Koller M, et al. Environmental toxicology and risk assessment of pharmaceuticals from hospital wastewater[J]. Water Research, 2011, 45(1): 75-92.

11. Thomas K V, Hilton M J. The occurrence of selected human pharmaceutical compounds in UK estuaries[J]. Marine Pollution Bulletin, 2004, 49(5/6): 436-444.

12. Hof H. Critical Annotations to the Use of Azole Antifungals for Plant Protection[J]. Antimicrobial Agents \& Chemotherapy, 2001, 45(11): 87-90.

13. David, Allen, Dustin, et al. Azole antifungals: 35 years of invasive fungal infection management: Expert Review of Anti-infective Therapy: Vol 13, No 6[J]. Expert Review of Anti Infective Therapy, 2015.13(6):787-798.

14. Wishart D S, Knox C, Guo A C, et al. DrugBank: a knowledgebase for drugs, drug actions and drug targets[J]. Nucleic Acids Research, 2007, 36(Database): D901-D906.

15. Autrup H. SCCP (Scientific Committee on Consumer Products) / SCHER (Scientific Committee on Health\& Environment Risks) / SCENIHR (Scientific Committee on Emerging and Newly-Identified Health Risks) opinion on:Risk assessment methodologies and approaches for genotoxic and carcinogenic substances[J].Radiographics A Review Publication of the Radiological Society of North America Inc, 2009, 22(2): e4.

16. Gouin T, Egmond R V, Price O R, et al. Prioritising chemicals used in personal care products in China for 
environmental risk assessment: Application of the RAIDAR model[J]. Environmental Pollution, 2012, 165(Jun.): 208-214.

17. Kathiravan M K, Salake A B, Chothe A S, et al. The biology and chemistry of antifungal agents: a review[J]. Bioorg Med Chem, 2012, 20(19): 56785698.

18. Roemer T, Krysan D J. Antifungal Drug Development: Challenges, Unmet Clinical Needs, and New Approaches[J]. Cold Spring Harbor Perspectives in Medicine, 2014, 4(5): a019703a019703.

19. Perlroth J, Choi B, Spellberg B. Nosocomial fungal infections: epidemiology, diagnosis, and treatment $[\mathrm{J}]$. Medical Mycology, 2007, 45(4): 321-346.

20. Wróbel T M, Kosikowska U, Kaczor A A, et al. Synthesis, Structural Studies and Molecular Modelling of a Novel Imidazoline Derivative with Antifungal Activity[J]. Molecules, 2015, 20(8): 6176.

21. Shafiei M, Peyton L, Hashemzadeh M, et al. History of the development of Antifungal azoles: A review on structures, SAR, and mechanism of action[J]. Bioorganic Chemistry, 2020: 104-240.

22. Jingxiang Zhou, Tao Yun, Quan Huang,et al. Comparative study on digestive enzyme activities of common carp, yellow croaker, mulberry and walleye[J]. Journal of Jilin Agricultural University, 2001, 23(1): 94-96.

23. Zhjiqiang Bian, Jin Zhang, Tao Wang, et al. Characteristics and mechanism of combined toxicity of carbamate pesticides to Chlorella pyrenoidosa [J]. Acta Ecotoxicology, 2019, 14(4): 150-162.

24. Renlong Cheng. Two-stage model predicts timedependent mixed toxicity of antibiotics to Chlorella pyrenoidea [D]. Anhui University of Architecture,2016. 